\title{
An investigation of atomic force microscopy, surface topography and adhesion of luting cements to zirconia: effect of silica coating, zirconia primer and laser
}

Yildirim, Bengisu ; Kümbüloğlu, Övül ; Saraçoğlu, Ahmet ; Al-Haj Husain, Nadin ; Özcan, Mutlu

\begin{abstract}
This study evaluated the effect various surface conditioning methods on the surface topography and adhesion of luting cements to zirconia. Zirconia blocks $(\mathrm{N}=25)$ were randomly assigned to five groups according to the surface conditioning methods: (a) No conditioning, control $(\mathrm{CON})$, (b) tribochemical silica coating (TSC), (c) MDP-based zirconia primer (ZRP), (d) coating with nano aluminum nitride (ALN) (e) etching with Er: YAG laser (LAS). The conditioned zirconia blocks were further divided into five subgroups to receive the luting cements: (a) MDP-based resin cement (Panavia F2.0) (PAN), (b) 4-META-based cement (Super Bond) (SUB), (c) UDMA-based (GCem) (GCE), (d) bis-GMA based (Bifix QM) (BIF) and (e) polycarboxylate cement (Poly-F) (POL). Cements were applied in polyethylene moulds (diameter: $3 \mathrm{~mm}$; height: $2 \mathrm{~mm}$ ). The bonded specimens were first thermocycled for 5500 cycles $\left(5-55{ }^{\circ} \mathrm{C}\right)$ and then adhesive interface was loaded under shear $(0.5 \mathrm{~mm} / \mathrm{min})$. The data $(\mathrm{MPa})$ were analyzed using 2-way ANOVA, Tukey's and Bonneferroni tests (alpha $=0.05)$. Regardless of the cement type, TSC resulted in significantly higher bond strength $(\mathrm{p} \quad 0.05)(13.3 \pm 4.35-25.3 \pm 6.3)$ compared to other conditioning methods $(2.96 \pm 1.5-5.4 \pm 5.47)$. Regardless of the surface conditioning method, no significant difference was found between MDP, 4-META and UDMA based cements ( $\mathrm{p}>0.05)$ being significantly higher than those of bis-GMA and polycarboxylate cements ( $\mathrm{p}$ 0.05). Failure types were frequently adhesive in all groups. Tribochemical silica coating provided superior bond results compared to other conditioning methods tested on zirconia especially in conjunction with UDMA- and 4-META-based resin cements.
\end{abstract}

DOI: https://doi.org/10.1080/01694243.2019.1620670

Posted at the Zurich Open Repository and Archive, University of Zurich

ZORA URL: https://doi.org/10.5167/uzh-183152

Journal Article

Accepted Version

Originally published at:

Yildirim, Bengisu; Kümbüloğlu, Övül; Saraçoğlu, Ahmet; Al-Haj Husain, Nadin; Özcan, Mutlu (2019). An investigation of atomic force microscopy, surface topography and adhesion of luting cements to zirconia: effect of silica coating, zirconia primer and laser. Journal of Adhesion Science and Technology, $33(18): 2047-2060$.

DOI: https://doi.org/10.1080/01694243.2019.1620670 
An investigation of atomic force microscopy, surface topography and adhesion of luting cements to zirconia: Effect of silica coating, zirconia primer and laser

\section{Bengisu Yildirim, DDS, $\mathrm{PhD}_{a}$ / Övül Kümbüloğlu, DDS, $\mathrm{PhD}_{\mathrm{b}}$ / Ahmet Saraçoğlu, DDS, $\mathrm{PhD}_{\mathfrak{b}}$ / Nadin Al-Haj Husain, MDent, Dr.med.dent.c / Mutlu Özcan, DDS, Dr.med.dent, Ph.Dd} aAssistant Professor, Usak University, Faculty of Dentistry, Department of Prosthodontics, Usak, Turkey aProfessor, Ege University, Faculty of Dentistry, Department of Prosthodontics, Izmir, Turkey cSpecialization Candidate, University of Bern, Department of Reconstructive Dentistry and Gerodontology, School of Dental Medicine, Bern, Switzerland aProfessor, University of Zurich, Dental Materials Unit, Center for Dental and Oral Medicine, Clinic for Fixed and Removable Prosthodontics and Dental Materials Science, Zurich, Switzerland

Short title: Adhesion of resin luting cements to zirconia

Correspondance to: Prof. Dr. med. dent. Mutlu Özcan, University of Zürich, Dental Materials Unit, Center for Dental and Oral Medicine Clinic for Fixed and Removable Prosthodontics and Dental Materials Science, Plattenstrasse 11, CH-8032, Zürich, Switzerland. Tel: +41-44-63 45600, e-mail: mutluozcan@hotmail.com 
Abstract: This study evaluated the effect various surface conditioning methods on the surface topography and adhesion of luting cements to zirconia. Zirconia blocks $(\mathrm{N}=25)$ were randomly assigned to 5 groups according to the surface conditioning methods: a) No conditioning, control (CON), b) tribochemical silica coating (TSC), c) MDP-based zirconia primer (ZRP), d) coating with nano aluminum nitride (ALN) e) etching with Er: YAG laser (LAS). The conditioned zirconia blocks were further divided into 5 subgroups to receive the luting cements: a) MDP-based resin cement (Panavia F2.0) (PAN), b) 4-META-based cement (Super Bond) (SUB), c) UDMA-based (GCem) (GCE), d) bis-GMA based (Bifix QM) (BIF) and e) polycarboxylate cement (Poly-F) (POL). Cements were applied in polyethylene moulds (diameter: $3 \mathrm{~mm}$; height: $2 \mathrm{~mm}$ ). The bonded specimens were first thermocycled for 5500 cycles $\left(5-55^{\circ} \mathrm{C}\right)$ and then adhesive interface was loaded under shear $(0.5 \mathrm{~mm} / \mathrm{min})$. The data (MPa) were analyzed using 2-way ANOVA, Tukey`s and Bonneferroni tests (alpha=0.05). Regardless of the cement type, TSC resulted in significantly higher bond strength $(p<0.05)(13.3 \pm 4.35-25.3 \pm 6.3)$ compared to other conditioning methods $(2.96 \pm 1.5-5.4 \pm 5.47)$. Regardless of the surface conditioning method, no significant difference was found between MDP, 4-META and UDMA based cements $(p>0.05)$ being significantly higher than those of bis-GMA and polycarboxylate cements $(p<0.05)$. Failure types were frequently adhesive in all groups. Tribochemical silica coating provided superior bond results compared to other conditioning methods tested on zirconia especially in conjunction with UDMAand 4-META-based resin cements.

Keywords: Adhesion; Air-abrasion; Laser; Resin cements; Shear bond strength; Silica-coating; Surface conditioning; Zirconia 


\section{Introduction}

For the clinical indications where aesthetic and high strength is of demand, zirconia is a good option for tooth or implant-borne reconstructions in dentistry. Zirconia is an inert biocompatible material with excellent mechanical properties due to stress-induced martensitic phase transformation [1-4]. Therefore, it is difficult to obtain sufficient bond adhesion of veneer and resin materials to zirconia [1-4]. Due to silica-free structure and high crystalline content, zirconia ceramics are resistant to hydrofluoric acid etching [5-7]. Therefore, in order to improve the bond strength of luting cements to zirconia, several physical and chemical surface conditioning methods have been proposed. These methods essentially aim for micro-mechanical, chemical or both micromechanical and chemical (physicochemical) interactions with the resin luting cement [8].

Airborne particle abrasion using $\mathrm{Al}_{2} \mathrm{O}_{3}$ particles is the most commonly employed physical conditioning method that promotes the bond strength between zirconia and luting cements. This method cleans the surface, increases the wettability and surface area. Also, micro-mechanical bonding is achieved with roughened surface [9]. However, airborne particle abrasion reduces the biaxial bending strength of the zirconia [10]. As a physicochemical surface conditioning method, tribochemical silica coating combined with silane coupling agents has been commonly advised for zirconia $[5,11,12]$. One other surface roughening method is the use of laser which may have consequences on the mechanical properties of zirconia depending on the laser parameters [13]. Recently, non-invasive methods like nano-structured alumina coating or primer applications have been advised for chemical activation $[14,15]$ that do not to cause micro cracks when a surface exposed roughening procedure[16]. One of the non-invasive conditioning methods for is application of nano-structured alumina coating that provides an active surface in order to promote bonding with luting cements [14]. Likewise, application of 10-Methacryloyloxydecyl dihydrogen phosphate (MDP) based primer is considered a non-invasive and easy method that increases the bond strength due to stable Zr-O-P bonds between zirconia and MDP [17]. 
In addition to the surface conditioning methods, adhesive resin cements based on MDP, 4Methacryloxyethyl trimellitate anhydride (4-META), urethane dimethacrylate (UDMA), or bisphenol A diglycidyl methacrylate (bis-GMA) play a significant role in adhesion to zirconia. While MDP and 4-META show better hydrolytic stability [12,18-21], self-adhesive resin cements do not require any surface conditioning to both the tooth and zirconia surface [21]. Although resin cements are advocated for bonding zirconia reconstructions, in some clinical studies, conventional cements showed adequate initial clinical stability [22, 23]. However, long term prognosis of retention was not sufficient [24]. Nonetheless, even though a great number of studies evaluated different surface conditioning methods and luting cements, a reliable bonding protocol for zirconia has not been defined to date and clinical studies show unfavourable results over 10 years of clinical service [25].

The objective of this study therefore was to evaluate the effect of physicochemical (invasive) and chemical (non-invasive) surface conditioning methods on the adhesion of resin-based and conventional cements to zirconia. The null hypothesis tested was that the various surface conditioning methods in combination with rein cements would not affect the bond strength to zirconia.

\section{Materials and Methods}

The materials used in this study and their chemical compositions are listed in Table 1.

\section{Specimen preparation}

Zirconia blocks $(\mathrm{N}=25)$ were obtained from pre-sintered zirconia (AvaDent Zirconia, NexxZR, Sagemax Bioceramics, Federal Way, WA, U.S.A). After sintering according to the manufacturer's instructions, the blocks measured approximately $21 \times 21 \times 10 \mathrm{~mm}$. Five blocks were used for each surface conditioning (5 types) and cements (5 types) combinations onto which 4 specimens were bonded yielding to 20 bonded specimens in each group ( $\mathrm{n}=20$ per group) (Fig. 1). 
The bonding surfaces of zirconia blocks were finished manually with wet 600-1200 grit silicon abrasive paper in sequence (SiC paper, Piramit, Istanbul, Turkey) and then ultrasonically cleaned (Quantrex 90, L\&R Ultrasonics, Kearny, NJ, USA) in water for 5 minutes.

Zirconia blocks were randomly assigned to 5 groups according to the surface conditioning methods:

\section{Surface conditioning methods}

Control (CON): In this group, zirconia surfaces were not conditioned.

Tribochemical silica coating (TSC): Zirconia surfaces were first air-abraded using $110 \mu \mathrm{m}$ alumina particles (Rocatec Pre, 3M ESPE, St. Paul, USA) wand then with $110 \mu \mathrm{m}$ alumina particles coated with silica (Rocatec Plus, 3M ESPE) at 2.8 bar pressure from a distance of $10 \mathrm{~mm}$ for $15 \mathrm{~s}$. Conditioned surfaces were silanized with silane coupling agent (ESPE-Sil, 3M ESPE) and waited for its reaction for 5 minutes.

Zirconia primer (ZRP): MDP-based zirconia primer (Z-Prime Plus, Bisco, Schaumburg, IL, U.S.A ) was applied with a micro brush onto the bonding surfaces of zirconia, gently dried for $10 \mathrm{~s}$ and then photopolymerized (Bluephase, Ivoclar Vivadent, Liechtenstein; light intensity: $1000 \mathrm{~mW} / \mathrm{cm}_{2}$ ) for $20 \mathrm{~s}$.

Coating with nano aluminum nitride (ALN): In this group, nano aluminum nitride powder (1.2 $\mu \mathrm{m}$ particle size, surface area: $6 \mathrm{~m} 2 / \mathrm{g}$, oxygen content: 2.5\%) (AIN Grade C, H.C. Stark, Munich, Germany) was applied on the bonding surface. AlN powder of $7.5 \mathrm{~g}$ was dispersed in $250 \mathrm{ml}$ preheated $\left(75^{\circ} \mathrm{C}\right.$ for 2 minutes) deionized water. Then, zirconia specimens were immersed in the suspension for 15 minutes, air-dried in an oven for 2 h. Lastly, they were heat treated in a furnace at $900^{\circ} \mathrm{C}$ for $1 \mathrm{~h}$.

Etching with Er: YAG laser (LAS): In group LAS, bonding surfaces of zirconia were irradiated by Er: YAG laser (Fotona, Ljubljana, Slovenia). Laser energy was delivered from a distance of $1 \mathrm{~mm}$ at $200 \mathrm{~mJ}, 200$ Watt, for $15 \mathrm{~s}$ under water cooling.

The conditioned zirconia blocks were further divided into 5 subgroups to receive the luting cements $(n=8$ per group): a) MDP-based resin cement (Panavia F2.0 Kuraray, Tokyo, Japan) (PAN), b) 4-META-based resin cement (Super Bond, C\&B, Sun Medical, Chiba, Japan) (SUB), c) UDMA-based resin cement (GCem, GC, 
Tokyo, Japan) (GCE), d) bis-GMA based resin cement (Bifix QM, Voco, Cuxhaven, Germany) (BIF) and e) polycarboxylate cement (Poly-F, Dentsply Sirona, Virum, Denmark) (POL).

\section{Bonding procedures and aging}

Polyethylene moulds with an internal diameter of $3 \mathrm{~mm}$ and a height of $2 \mathrm{~mm}$ were placed perpendicular on the bonding surfaces of zirconia blocks (Fig. 1). Except for groups SUB and POL, the specimens were photopolymerized for $40 \mathrm{~s}$ from each side (Bluephase, light intensity: $1000 \mathrm{~mW} / \mathrm{cm}_{2}$ ) and oxygen-inhibiting gel (Oxyguard II, Kuraray) was applied on the free surfaces of resin cement on zirconia specimens.

After bonding, specimens were thermocycled between 5 and $55^{\circ} \mathrm{C}$ for 5500 cycles with a dwell of time $20 \mathrm{~s}$ (Smart RoboTechnologies, Esetron, Ankara, Turkey).

\section{Bond strength test}

The bonded specimens were fixed in the jig of the universal testing machine (Shimadzu, Kyoto, Japan) and the bonded interface was subjected to shear loading at a crosshead speed of $0.5 \mathrm{~mm} / \mathrm{min}$ (Fig. 2). Shear bond strength (SBS) was calculated in MPa by dividing the maximum load F (Newton) to debond the cement by the bonded surface area $(\mathrm{S})\left(\mathrm{mm}_{2}\right)$ using the SBS=F/S formula.

After debonding, failure types were analyzed from all specimens under optical microscope (Leica microsytems, Leica, Wetzlar, Germany) at x10 magnification. Failure types were classified as: a) adhesive failure at the zirconia-resin interface with no resin remnants on the substrate, b) mixed (adhesive failure at the at the zirconia-resin interface and cohesive failure in the cement), c) cohesive failure in the cement.

The specimens that have demonstrated the highest and the lowest values from each subgroup were selected and further analyzed under a Scanning Electron Microscope (SEM) (ESEM, Quanta 250 FEG, Oregon, USA) at x5000 magnification.

According to the bond strength test results, the highest bond strength value was measured in group TSC and the lowest in group CON. Therefore, the surface roughness of TSC and CON was analyzed in separate conditioned ( $\mathrm{n}=3$ per group) specimens with Atomic Force Microscopy (AFM) Multimode Nanoscope IV, 
Digital Instruments Veeco Metrology Group, Plainview, NY, USA) at $0.1 \mathrm{~Hz}$. Surface roughness (Ra) was measured at three random locations $(\mu \mathrm{m})$.

\section{Statistical analysis}

Statistical analysis was performed using SPSS 18.0 software (SPSS Inc., Chicago, IL, USA). Shapiro-Wilk and Levene tests were used to test normal distribution of the data. As the data were not homogeneously distributed, SBS data were analyzed using 2-way ANOVA, Tukey`s test and Bonferroni post-hoc corrections where the bond strength was the dependent variable and conditioning methods ( 5 levels: CON, TSC, ZRP, ALN, LAS) and resin cements (5 levels: PAN, SUB, GCE, BIF, POL) as independent variables. Ra values were analysed using Kolmogorov-Smirnov test followed by Kruskal-Wallis test. P values less than 0.05 were considered to be statistically significant in all tests.

\section{Results}

Bond strength of the luting cements to zirconia were significantly affected by the surface conditioning method $(p<0.05)$ and the luting cement type $(p<0.05)$. Interaction terms were also significant $(p<0.05)$.

Regardless of the cement type, TSC method resulted in significantly higher bond strength with the resin luting cements $(13.30 \pm 4.35$ - 25.31 \pm 6.27$)$ than those with other conditioning methods $(2.96 \pm 1.54$ $15.42 \pm 5.47)(p<0.05)($ Table 2$)$. In the CON group, PAN $(10.28 \pm 4.28)$ and SUB $(7.67 \pm 1.4)$ cements showed significantly higher results compared to other cements $(1.27 \pm 0.29-6.99 \pm 1.63)$. TSC provided superior bond results compared to other conditioning methods especially in conjunction with GCE $(25.31 \pm 6.27)$.

Regardless of the surface conditioning method, no significant difference was found between PAN, SUB and GCE based cements ( $p>0.05)$ being significantly higher than those of BIF and POL cements $(p<0.05)$.

Failure types were predominantly adhesive in all groups (Table 3). Only in group SUB, cohesive failures in the cement was more frequently observed than with other cement groups. 
SEM images indicated surface morphologies at varying levels depending on the conditioning method where ZRP and AIN showed smoother surfaces (Figs. 3a-e). The surface roughness (Ra) results measured using AFM revealed significantly higher roughness after TSC $(83 \pm 35.4 \mu \mathrm{m})$ compared to CON $(45.8 \pm 20 \mu \mathrm{m})$ $(p<0.05)$. (Figs. 4a-b).

\section{Discussion}

This study investigated the effect of physicochemical (invasive) and chemical (non-invasive) surface conditioning methods on the adhesion of resin-based and polycarboxylate cements to zirconia. Since bond strength of the luting cements to zirconia were significantly affected by the surface conditioning method (and the luting cement type, the null hypothesis could be rejected.

One of the most commonly employed physicochemical surface conditioning method for zirconia is tribochemical silica coating. With this method however, particle size and deposition duration were reported to cause micro cracks in zirconia impairing its mechanical stability $[7,18,19]$. Furthermore, wettability of the silane coupling agent into the deep grooves after air-abrasion may not be ideal [18]. Nevertheless, mechanical and chemical benefits of tribochemical silica coating increases the adhesion of resin cements to zirconia depending in the cement type [11,26-31]. On the other hand, silica coating may not always result in a uniform distribution of silica layer on the zirconia surfaces and the chemical bonding with silane coupling agent is prone to hydrolytic degradation $[5,9,32,33]$, resulting in decrease in adhesion of resin cements to zirconia after artificial aging. Özcan et al reported that MDP based resin cements combined with physicochemical surface conditioning methods provide more durable adhesion as opposed to self-adhesive resin cements [8]. In this study, after tribochemical silica coating, the highest value was obtained in the selfadhesive cement GCE (25.31 MPa) being significantly higher than with MDP-based cement PAN (13.3 MPa).

Similar to our results, in a previous study [21] with GCE 22.4 MPa bond strength was reported after air abrasion. For group ROC-PAN combination, SBS values in previous other studies [34-36] ranged between 
26.1-27.2 MPa, where microtensile bond strength was used after 7 days of after storage in distilled water at $37^{\circ} \mathrm{C}$. The lower results in this study, could be due to the selection of a different test method and application of long term thermocycling.

The zirconia primers containing MDP monomer were reported to provide reliable adhesion between resin cements and zirconia [37-40]. Application of such primers as a chemical conditioning method eliminates possible damage created on zirconia after air-abrasion. Zirconia Prime Plus contains MDP, BPDMA HEMA organophosphate monomer and carboxylic acid monomers [15]. Phosphate-monomer group has an affinity to the metal oxides of zirconia and forms a chemical adhesion through hydrogen bonds $[17,38]$. Thermocycling however, decreases the bond strength achieved with the hydroxyl groups of primers $[18,39,40]$. Therefore, when primers are used, application of long-term thermal cycling is suggested [18]. In this study, MDP containing Z-Primer increased the shear bond strength values with the resin cements significantly especially in ZPR-PAN group with $15.42 \mathrm{MPa}$. In another study, with the same combination bond strength of $14.96 \mathrm{MPa}$ was reported [15]. In the absence of air-abrasion, where micro-mechanical interlocking is missing, chemical adhesion and its hydrolytic stability through MDP containing primers is crucial. In this study, wettability measurements were not performed which could be considered as a limitation of the study. Since good wettability is essential for reliable adhesion, future studies should also focus on the wettability properties of the selected zirconia primer and the cements.

One other non-invasive surface conditioning method with nano-structured alumina coating was proposed to activate the zirconia surface that increases the surface area and affects the mechanical and chemical bonding [14]. Moreover, it was claimed that alumina coating effectively modifies the cementation surface of zirconia. [41]. The results of this study are in agreement with this study but contradicts with another study where ALN coating did not positively affect the bond strength of resin cements to zirconia [39]. According to this study, ALN in combination with GCE resin cement showed significantly higher values than those of other cements indicating that not only the conditioning but the resin cement type dictates durable adhesion. With 
ALN-PAN combination, Zhang et al found 32.6 to $44.5 \mathrm{MPa}$ tensile bond strength [42] while in this study ALNPAN resulted in $10.07 \mathrm{MPa}$. It was supposed that the different test methods are the reason for the difference. Er:YAG laser etching method did not always result in strong bond [38] and applying high concentration (400-600 mJ) caused more material loss and formation of deep cracks than with low concentration (200 mJ) and therefore the latter was suggested for better adhesion to zirconia [43]. Cavalcanti et al [44] found 15.8 MPa micro shear bond strength value for PAN and 3 MPa for Calibra (Bis-GMA based) cement after Er:YAG laser conditioning on zirconia surface. In this study, Er:YAG laser was preferred $s$ it is a hard tissue laser, which was irradiated at $200 \mathrm{~mJ}$ with constant water cooling. Laser etching combined with PAN, GCE and SUB significantly increased the bond strength compared to POL and BIF. Nevertheless, in this and a previous study [45] Er:YAG laser irradiation was not as effective as tribochemical silica coating for improved adhesion to zirconia. Although some studies showed that cement selection was more important than surface conditioning method for retention of zirconia crowns [46], Gomes et al [13] reported that surface conditioning is more essential in zirconia-cement bonding. In addition, Özcan et al [8] stated that surface conditioning method in combination with the resin cement type has a significant effect on bond strength values to zirconia. In this study, conventional cement presented the lowest results regardless of the conditioning method. In other studies, zinc phosphate, glass ionomer and resin cements showed no significant difference when compared to MDP containing resin cement $[47,48,49]$. This is most probably due to the lack of thermocycling that drastically decreases adhesion values achieved with glass-ionomer cements [11]. Likewise, in this study, the specimens in the conventional cement groups failed exclusively adhesive indicating that chemical bonding is essential for zirconia cementation.

Chemical composition, wetting capability, viscosity, and mechanical properties of cements may also affect the bond strength to zirconia [50]. In present study, resin cement selection has been made according to their composition. While MDP monomer containing cements improve the bond strength to zirconia [5,51], chemical reaction occurs between zirconia hydroxyl groups and phospate esther monomers of MDP [12]. Oyagüe et al 
[52] stated that MDP containing cements may be used without employing any surface conditioning method. However, Özcan et al reported that after thermocycling MDP based resin cement bond strength values decrease dramatically [53]. In group CON, where no surface conditioning was applied, group PAN exhibited significantly higher bond strength compared to POL and BIF. On the contrary, De Souza et al [54] reported that without surface conditioning, MD-based PAN does not increase the bond strength.

On other resin cement studies was SUB which has no inorganic fillers and consists of 4-META/MMA-TBB resin. It was concluded pre-treatment of zirconia surface improve the bonding efficiency of 4-META/MMATBB resin [55]. This chemically polymerized resin cement with its long flexible chains and high molecular weight has high fracture toughness values [56]. While Ernst et al [57] found 4.8 MPa retentive strength, Derand et al [6] reported the highest bond strength value with SUB regardless of the surface conditioning method. In this study, for group CON-SUB bond strength of 7.67 MPa was obtained which was similar to results of Moon et al [58] with 8.6 MPa. Similarly, 4-META and UDMA are the main monomers of GCE. While 4-META forms a chemical reaction with metal oxides [21], UDMA may suffer from water absorption in hydrophilic methacrylate, resulting in decrease in adhesion after thermocycling. On the other hand, although bis-GMA based resin cements have sufficient bond strength value after silica containing ceramics, it is not a suitable cement type for zirconia as it does not contain functional monomer [5,6]. Accordingly, BIF cement exhibited significantly lower bond strength values than other resin adhesive cements. Also, in this study, adhesive failure types were more frequently observed which signifies the weak intermolecular affinity between cement polymer or monomer functional pole and zirconia hydroxyl groups [29,54,59].

Shear bond test causes non-homogenous stress distribution when performed with dentin or glass-ceramic and predominantly cohesive failure was reported [60,61]. According to Valandro et al [35], shear bond test is more appropriate testing adhesion to high strength ceramics. Since zirconia is a tough ceramic, cohesive failures in the zirconia ceramic is almost impossible to achieve. Furthermore, Özcan et al [8] stated that adhesive interface is exposed to both tensile and shear forces in intraoral conditions. Therefore, there is no 
recommendation as to which test method can be used. In summary, increasing the surface roughness of zirconia surface would help clean the surface and increase micro-mechanical retention which could also be verified with the SEM and SEM findings but the resin cement type has also a significant role in obtaining durable adhesion to zirconia.

\section{Conclusions}

From this study, the following could be concluded:

1. Conventional polycarboxylate cement cement exhibited the lowest bond strength to zirconia after all conditioning methods due to the lack of chemical bonding.

2. With all resin cements tested, tribochemical silica coating presented the highest bond strength to zirconia exceeding $10 \mathrm{MPa}$ and the highest roughness values compared to other conditioning methods.

3. Tibochemical silica coating resulted in the highest bond strength values with UDMA (G-Cem) and 4META containing (Super Bond) cements.

4. Irrespective of the surface conditioning method, mean bond strength obtained with MDP, 4-META and UDMA based cements were significantly higher than those of bis-GMA and polycarboxylate cements.

5. Failure types were frequently adhesive in all groups.

\section{Clinical Relevance}

Adhesion of resin cements with different chemistries to zirconia could be improved after tribochemical silica coating compared to zirconia primer alone, coating with nano aluminum nitride or laser applications. While UDMA, 4-META and MDP based cements performed better, failure types were still mainly adhesive.

\section{Conflict of interest}

The authors did not have any commercial interest in any of the materials used in this study. 


\section{References}

[1] Chevalier J, Gremillard L, Virkar AV, et al. The tetragonal-monoclinic transformation in zirconia: lessons learned and future trends. J. Amer. Ceramic Soc. 2009;92:1901-1920.

[2] Kosmač $T$, Oblak $C$, Jevnikar $P$, et al. The effect of surface grinding and sandblasting on flexural strength and reliability of Y-TZP zirconia ceramic. Dent. Mater. 1999;15:426-433.

[3] Piconi C, Maccauro G. Zirconia as a ceramic biomaterial. Biomaterials 1999;20:1-25.

[4] Emanuele C, Cosmin S, Antoniac, I et al. Shear bond strength tests of zirconia veneering ceramics after chipping repair and to the adhesive cements. J. Adhes. Sci. Technol. 2016:30:1-11.

[5] Kern M, Wegner SM. Bonding to zirconia ceramic: adhesion methods and their durability. Dent. Mater. $1998 ; 14: 64-71$.

[6] Dérand P, Dérand T. Bond strength of luting cements to zirconium oxide ceramics. Int. J. Prosthodont. 2000;13:131-135.

[7] Özcan M, Vallittu PK. Effect of surface conditioning methods on the bond strength of luting cement to ceramics. Dent. Materials. 2003;19:725-731.

[8] Özcan M, Bernasconi M. Adhesion to zirconia used for dental restorations: a systematic review and metaanalysis. J. Adhes. Dent. 2015;17:7-26.

[9] Blatz MB, Sadan A, Kern M. Resin-ceramic bonding: a review of the literature. J. Prosthet. Dent. 2003;89:268-274.

[10] Kosmac T, Oblak C, Jevnikar P, et al. Strength and reliability of surface treated Y-TZP dental ceramics. J Biomed Mater Res. 2000;53:304-313.

[11] Piwowarczyk A, Lauer H, Sorensen J. The shear bond strength between luting cements and zirconia ceramics after two pre-treatments. Oper. Dent. 2005;30:382.

[12] Thompson JY, Stoner BR, Piascik JR, et al. Adhesion/cementation to zirconia and other non-silicate ceramics: where are we now? Dent. Mater. 2011;27:71-82. 
[13] Gomes AL, Ramos JC, Santos-del Riego S, et al. Thermocycling effect on microshear bond strength to zirconia ceramic using Er: YAG and tribochemical silica coating as surface conditioning. Lasers Med. Sci. $2015 ; 30: 787-795$.

[14] Jevnikar P, Krnel K, Kocjan A, et al. The effect of nano-structured alumina coating on resin-bond strength to zirconia ceramics. Dent. Mater. 2010;26:688-696.

[15] Magne P, Paranhos MP, Burnett LH. New zirconia primer improves bond strength of resin-based cements. Dent. Mater. 2010;26:345-352.

[16] Aboushelib MN, Kleverlaan CJ, Feilzer AJ. Selective infiltration-etching technique for a strong and durable bond of resin cements to zirconia-based materials. J. Prosthet. Dent. 2007;98:379-388.

[17] Pilo R, Kaitsas V, Zinelis S, et al. Interaction of zirconia primers with yttria-stabilized zirconia surfaces. Dent. Mater. 2016;32:353-362.

[18] Özcan M, Nijhuis H, Valandro LF. Effect of various surface conditioning methods on the adhesion of dual-cure resin cement with MDP functional monomer to zirconia after thermal aging. Dent. Mater. J. 2008;27:99-104.

[19] Oyagüe RC, Monticelli F, Toledano M, et al. Effect of water aging on microtensile bond strength of dualcured resin cements to pre-treated sintered zirconium-oxide ceramics. Dent. Mater. 2009;25:392-399.

[20] de Oyagüe RC, Monticelli F, Toledano M, et al. Influence of surface treatments and resin cement selection on bonding to densely-sintered zirconium-oxide ceramic. Dent. Mater. 2009;25:172-179.

[21] Blatz MB, Phark J-H, Ozer F, Mante FK, et al. In vitro comparative bond strength of contemporary selfadhesive resin cements to zirconium oxide ceramic with and without air-particle abrasion. Clin. Oral Invest. $2010 ; 14: 187-192$.

[22] Von Steyern PV, Carlson P, Nilner K. All-ceramic fixed partial dentures designed according to the DCZirkon® technique. A 2-year clinical study. J. Oral Reahbil. 2005;32:180-187. 
[23] Tinschert J, Schulze KA, Natt G, et al. Clinical behavior of zirconia-based fixed partial dentures made of DC-Zirkon: 3-year results. Int. J. Prosthodont. 2008;21;217-222.

[24] Uo M, Sjögren G, Sundh A, et al. Effect of surface condition of dental zirconia ceramic (Denzir) on bonding. Dent. Mater. J. 2006;26:626-631.

[25] Rathmann F, Bömicke W, Rammelsberg $P$, et al. Veneered zirconia inlay-retained fixed dental prosthesis:10-year results from a prpspective clinical study. J. Dent. 2017;64:68-72.

[26] Atsu SS, Kilicarslan MA, Kucukesmen HC, et al. Effect of zirconium-oxide ceramic surface treatments on the bond strength to adhesive resin. J. Prosthet. Dent. 2006;95:430-436.

[27] Blatz MB, Chiche G, Holst S, et al. Influence of surface treatment and simulated aging on bond strengths of luting agents to zirconia. Quint. Int. 2007;38:745-753.

[28] Amaral R, Özcan M, Valandro LF, et al. Effect of conditioning methods on the microtensile bond strength of phosphate monomer-based cement on zirconia ceramic in dry and aged conditions. J. Biomed. Mater. Res. Part B: Appl. Biomaterials. 2008;85:1-9.

[29] Tanaka R, Fujishima A, Shibata Y, et al. Cooperation of phosphate monomer and silica modification on zirconia. J. Dent. Res. 2008;87:666-670.

[30] Mahmoodi N, Hooshmand T, Heidari S, et al. Effect of sandblasting, silica coating, and laser treatment on the microtensile bond strength of a dental zirconia ceramic to resin cements. Lasers Med. Sci. 2016;31:205-211.

[31] Ranjbar OB, Karimi YP, Oveisi, et al. Comparison of Micro-Shear Bond Strength of Resin Cement to Zirconia With Different Surface Treatments Using Universal Adhesive and Zirconia Primer. J. Lasers Med. Sci. 2018;9:200-206.

[32] Wegner SM, Kern M. Long-term resin bond strength to zirconia ceramic. J. Adhes. Dent. 2000;2:139147. 
[33] Matinlinna JP, Heikkinen T, Özcan M, et al. Evaluation of resin adhesion to zirconia ceramic using some organosilanes. Dent. Mater. 2006;22:824-831.

[34] Bottino MA, Valandro LF, Scotti R, et al. Effect of surface treatments on the resin bond to zirconiumbased ceramic. Int. J. Prosthodont. 2005;18:60-65.

[35] Kumbuloglu O, Lassila L, User A, et al. Bonding of resin composite luting cements to zirconium oxide by two air-particle abrasion methods. Oper. Dent. 2006;31:248-255.

[36] Valandro LF, Özcan M, Amaral R, et al. Effect of testing methods on the bond strength of resin to zirconia-alumina ceramic: microtensile versus shear test. Dent. Mater. J. 2008;27:849-855.

[37] Kitayama S, Nikaido T, Takahashi R, et al. Effect of primer treatment on bonding of resin cements to zirconia ceramic. Dent. Mater. 2010;26:426-432.

[38] Foxton RM, Cavalcanti AN, Nakajima M, et al. Durability of resin cement bond to aluminium oxide and zirconia ceramics after air abrasion and laser treatment. J. Prosthodont. 2011;20:84-92.

[39] Külünk T, Külünk Ş, Baba $S$, et al. The effect of alumina and aluminium nitride coating by reactive magnetron sputtering on the resin bond strength to zirconia core. J Adv. Prosthod. 2013;5:382-387.

[40] Yang L, Xie H, Meng H, et al. Effects of of luting cements and surface conditioning on composite bonding performance to zirconia. J Adhes Dent 2018;20:549-558.

[41] Srikanth R, Kosmac T, Della Bona A, et al. Effects of cementation surface modifications on fracture resistance of zirconia. Dent. Mater. 2015;31:435-442.

[42] Zhang S, Kocjan A, Lehmann F, et al. Influence of contamination on resin bond strength to nanostructured alumina-coated zirconia ceramic. Eur. J. Oral. Sci. 2010;118:396-403.

[43] Cavalcanti AN, Pilecki P, Foxton RM, et al. Evaluation of the surface roughness and morphologic features of Y-TZP ceramics after different surface treatments. Photomed. Laser Surg. 2009;27:473-479.

[44] Cavalcanti AN, Foxton RM, Watson TF, et al. Bond strength of resin cements to a zirconia ceramic with different surface treatments. Oper. Dent. 2009;34:280-287. 
[45] Akin H, Tugut F, Akin GE, et al. Effect of Er: YAG laser application on the shear bond strength and microleakage between resin cements and Y-TZP ceramics. Lasers Med. Sci. 2012;27:333-338.

[46] Karimipour-Saryazdi M, Sadid-Zadeh R, Givan D, et al. Influence of surface treatment of yttriumstabilized tetragonal zirconium oxides and cement type on crown retention after artificial aging. J. Prosthet. Dent. 2014;111:395-403.

[47] Kappert HF. Keramiken-eine Ubersicht. Quintessenz Zahntech. 2001;2:668-704.

[48] Uo M, Sjoren G, Sundh A, et al. Cytotoxicity and bonding property of dental ceramics. Dent. Mater. $2003 ; 19: 487-492$.

[49] Lüthy $\mathrm{H}$, Loeffel O, Hammerle $\mathrm{CH}$. Effect of thermocycling on bond strength of luting cements to zirconia ceramic. Dent. Mater. 2006;22:195-200.

[50] Attia A. Bond strength of three luting agents to zirconia ceramic-influence of surface treatment and thermocycling. J. Appl. Oral Sci. 2011;19:388-395.

[51] Koizumi H, Nakayama D, Oba Y, et al. Effect of acidic primers on adhesive bonding of tri-n-butylborane initiated adhesive resin to alumina. J. Oral Sci. 2010;52:571-576.

[52] Oyagüe RC, Osorio R, da Silveira BL, et al. Comparison of bond stability between dual-cure resin cements and pretreated glass-infiltrated alumina ceramics. Photomed. Laser Surg. 2011;29:465-475.

[53] Özcan M, Kerkdijk S, Valandro LF. Comparison of resin cement adhesion to Y-TZP ceramic following manufacturers' instructions of the cements only. Clin. Oral. Invest. 2008;12:279-282.

[54] de Souza GMD, Silva NR, Paulillo LA, et al. Bond strength to high-crystalline content zirconia after different surface treatments. J. Biomed. Mater. Res. Part B: Appl. Biomaterials. 2010;93:318-323.

[55] Shimizu H, Inokoshi M, Takagaki $T$, et al. Bonding efficacy of 4-META/MMA-TBB resin to surfacetreated highly translucent dental zirconia. J. Adhes. Dent. 2018;20:453-459.

[56] Komine F, Tomic M, Gerds T, et al. Influence of different adhesive resin cements on the fracture strength of aluminum oxide ceramic posterior crowns. J. Prosthet. Dent. 2004;92:359-364. 
[57] Ernst C-P, Cohnen U, Stender E, et al. In vitro retentive strength of zirconium oxide ceramic crowns using different luting agents. J. Prosthet. Dent. 2005;93:551-558.

[58] Moon J-e, Kim S-h, Lee J-b, et al. The effect of preparation order on the crystal structure of yttriastabilized tetragonal zirconia polycrystal and the shear bond strength of dental resin cements. Dent. Mater. $2011 ; 27: 651-663$.

[59] Mirmohammadi H, Aboushelib MN, Salameh Z, et al. Innovations in bonding to zirconia based ceramics: Part III. Phosphate monomer resin cements. Dent. Mater. 2010;26:786-792.

[60] Della Bona A, Van Noort R. Shear vs. tensile bond strength of resin composite bonded to ceramic. J. Dent. Res. 1995;74:1591-1596.

[61] Braga RR, Meira JB, Boaro LC, et al. Adhesion to tooth structure: a critical review of "macro" test methods. Dent. Mater. 2010;26:e38-e49. 


\section{Captions to figures and tables:}

\section{Tables:}

Table 1 Brands, abbreviations, chemical compositions and manufacturers of the materials used in this study. Table 2 Mean shear bond strength values (MPa \pm Standard Deviations) of experimental groups. *Uppercase letters in one column represents statistically significant differences based on conditioning method and lowercase letters based on cement types $(p<0.05)$. See Table 1 for group abbreviations.

Table 3 Distribution of failure types in each experimental group: A: Adhesive failure at the zirconia-resin interface with no resin remnants on the substrate, C: Cohesive failure in the cement, M: Mixed failure: adhesive failure at the at the zirconia-resin interface and cohesive failure in the cement.

\section{Figures:}

Fig. 1 Dimensions of the substrate zirconia specimen and the resin cements bonded using polyethylene moulds.

Fig. 2 Schematic drawing of the specimen in the jig for the universal testing machine subjected to shear bond strength test.

Figs. 3a-e SEM images of zirconia surfaces $(x 5000)$ a) Control, b) Tribochemical silica coating, c) Zirconia primer, d) Nano aluminium nitride coating, e) Laser irradiation. Note that surface morphologies varied depending on the conditioning method where zirconia primer and nano aluminium nitride coating showed smoother surfaces.

Figs. 4a-b AFM images of a) Control, non-conditioned $(45.8 \pm 20 \mu \mathrm{m})$, b) Tribochemical silica coated $(83 \pm 35.4$ $\mu \mathrm{m})$ zirconia surfaces. 

Tables:

\begin{tabular}{l|l|l}
\hline Material & \multicolumn{1}{|c}{ Chemical Composition } & Manufacturer \\
\hline $\begin{array}{l}\text { Poly F } \\
\text { (POL) }\end{array}$ & $\begin{array}{l}\text { Powder: Zinc polycarboxylate } \\
\text { Liquid: Zinc polycarboxylate acid }\end{array}$ & Dentsply, Virum, Denmark \\
\hline $\begin{array}{l}\text { Panavia F 2.0 } \\
\text { (PAN) }\end{array}$ & $\begin{array}{l}\text { Paste A: MDP, DMA, silanated silica filler } \\
\text { silanated colloidal silica, dl-Camphorquinone } \\
\text { Paste B: DMA, pigments, accelerators }\end{array}$ & Kuraray, Tokyo, Japan \\
\hline $\begin{array}{l}\text { Super Bond } \\
\text { (SUB) }\end{array}$ & 4-META, TBB, MMA & Sun Medical, Chiba, Japan \\
\hline $\begin{array}{l}\text { G-Cem } \\
\text { (GCE) }\end{array}$ & UDMA, camphorquinone, hydroperoxide & GC, Tokyo, Japan \\
\hline $\begin{array}{l}\text { Bifix QM } \\
\text { (BIF) }\end{array}$ & bis-GMA, benzoylperoxide, amin & Voco, Cuxhaven, Germany \\
$\begin{array}{l}\text { Z-Prime Plus } \\
\text { (ZRP) }\end{array}$ & MDP, bis-GMA, organophosphate, carboxylic acid & Bisco, Schaumburg, USA \\
\hline $\begin{array}{l}\text { Rocatec Pre } \\
\text { (TSC) }\end{array}$ & $110 \mu \mathrm{m}$ Al2O3 particles & $3 \mathrm{M}$ ESPE, St. Paul, USA \\
\hline $\begin{array}{l}\text { Rocatec Plus } \\
\text { (TSC) }\end{array}$ & $110 \mu \mathrm{m}$ silica coated Al2O3 particles & 3M ESPE \\
\hline $\begin{array}{l}\text { ESPE-Sil } \\
\text { Nano } \\
\text { aluminum } \\
\text { nitride } \\
\text { (ALN) }\end{array}$ & $\begin{array}{l}\text { 3-methacryloxyprophyltrimethoxy silane in ethanol } \\
\text { Surface area: } 6 \text { m2/g, O } \mathrm{O}_{2} \text { content: } 2.5 \text { wt\% }\end{array}$ & $3 \mathrm{~m}$ ESPE \\
\hline
\end{tabular}

Table 1 Brands, abbreviations, chemical compositions and manufacturers of the materials used in this study. 


\begin{tabular}{|c|c|c|c|c|}
\hline $\begin{array}{l}\text { Surface Conditioning } \\
\text { ( } n=8 \text { per group) }\end{array}$ & Cements & Mean \pm Standard Deviation (MPa) & Lower bound & Upper bound \\
\hline \multirow[t]{5}{*}{ CON } & PAN & $10.28 \pm 4.28 \mathrm{a}, \mathrm{A}, \mathrm{B}$ & 6.7 & 13.86 \\
\hline & SUB & $7.67 \pm 1.4 \mathrm{a}, \mathbf{b}, \mathbf{A}, \mathbf{C}$ & 5.49 & 7.85 \\
\hline & POL & $1.27 \pm 0.29 b, A$ & 0.8 & 1.73 \\
\hline & GCE & $6.99 \pm 1.63 \mathbf{a}, \mathbf{b}, \mathbf{A}$ & 5.62 & 8.36 \\
\hline & $\mathrm{BIF}$ & $2.75 \pm 1.18 b, A$ & 1.77 & 3,74 \\
\hline \multirow[t]{5}{*}{ TSC } & PAN & $13.30 \pm 4.35 a, A, B$ & 9.66 & 16.95 \\
\hline & SUB & $21.18 \pm 6.24 a, c, B$ & 15.96 & 26.41 \\
\hline & POL & $1.73 \pm 0.36 b, A$ & 1.28 & 2.17 \\
\hline & GCE & $25.31 \pm 6.27 c, B$ & 20.07 & 30.56 \\
\hline & $\mathrm{BIF}$ & $15.09 \pm 6.12 a, \mathbf{B}$ & 9.96 & 20.21 \\
\hline \multirow[t]{5}{*}{ ZRP } & PAN & $15.42 \pm 5.47 \mathrm{a}, \mathbf{A}$ & 10.85 & 20.00 \\
\hline & SUB & $13.73 \pm 6.42 \mathrm{a}, \mathbf{A}$ & 8.36 & 19.10 \\
\hline & POL & $2.24 \pm 1.13 b, A$ & 0.83 & 3.65 \\
\hline & GCE & $13.32 \pm 4.23 a, c$ & 9.77 & 16.86 \\
\hline & $\mathrm{BIF}$ & $9.34 \pm 2.76 a, b, c$ & 7.02 & 11.65 \\
\hline \multirow[t]{5}{*}{ ALN } & PAN & $5.64 \pm 1.86 a, c, B$ & 4.08 & 7.2 \\
\hline & SUB & $5.94 \pm 2.78 \mathrm{a}, \mathrm{C}$ & 3,61 & 8.27 \\
\hline & POL & $2.23 \pm 0.86 c, A$ & 2.09 & 2.37 \\
\hline & GCE & $10.07 \pm 1.5$ b.A,C & 8.65 & 11.5 \\
\hline & $\mathrm{BIF}$ & $6.62 \pm 1.29 \mathrm{a}, \mathrm{A}, \mathrm{C}$ & 5.54 & 7.71 \\
\hline \multirow[t]{5}{*}{ LAS } & PAN & $10.93 \pm 4.94 a, A, B$ & 6.8 & 15.06 \\
\hline & SUB & $9.33 \pm 4.93 a-A, C$ & 4.77 & 13.9 \\
\hline & $\mathrm{POL}$ & $2.03 \pm 1.76 b, \mathbf{A}$ & 0.68 & 3.39 \\
\hline & GCE & $11.50 \pm 4.51 \mathrm{a} . \mathrm{A}, \mathrm{C}$ & 7.77 & 15.31 \\
\hline & $\mathrm{BIF}$ & $2.96 \pm 1.54$ b,A,D & 1.67 & 4.26 \\
\hline
\end{tabular}


Table 2 Mean shear bond strength values (MPa \pm Standard Deviations) of experimental groups. *Uppercase letters in one column represents statistically significant differences based on conditioning method and lowercase letters based on cement types $(p<0.05)$. See Table 1 for group abbreviations.

\begin{tabular}{llllll} 
& CON & TBS & ZRP & AIN & LAS \\
\cline { 2 - 5 } & $\mathrm{A} / \mathrm{C} / \mathrm{M}$ & $\mathrm{A} / \mathrm{C} / \mathrm{M}$ & $\mathrm{A} / \mathrm{C} / \mathrm{M}$ & $\mathrm{A} / \mathrm{C} / \mathrm{M}$ & $\mathrm{A} / \mathrm{C} / \mathrm{M}$ \\
PAN & $6 / 1 / 1$ & $5 / 1 / 2$ & $5 / 1 / 2$ & $7 / 1 / 0$ & $4 / 3 / 1$ \\
SUB & $3 / 4 / 1$ & $4 / 4 / 0$ & $3 / 5 / 8$ & $3 / 4 / 1$ & $2 / 4 / 2$ \\
POL & $8 / 0 / 0$ & $8 / 0 / 0$ & $8 / 0 / 0$ & $8 / 0 / 0$ & $8 / 0 / 0$ \\
GCE & $6 / 2 / 0$ & $5 / 1 / 2$ & $4 / 3 / 1$ & $7 / 1 / 0$ & $7 / 0 / 1$ \\
BIF & $6 / 1 / 1$ & $7 / 1 / 0$ & $7 / 1 / 0$ & $8 / 0 / 0$ & $8 / 0 / 0$
\end{tabular}

Table 3 Distribution of failure types in each experimental group: A: Adhesive failure at the zirconia-resin interface with no resin remnants on the substrate, $\mathrm{C}$ : Cohesive failure in the cement, M: Mixed failure: adhesive failure at the at the zirconia-resin interface and cohesive failure in the cement. 


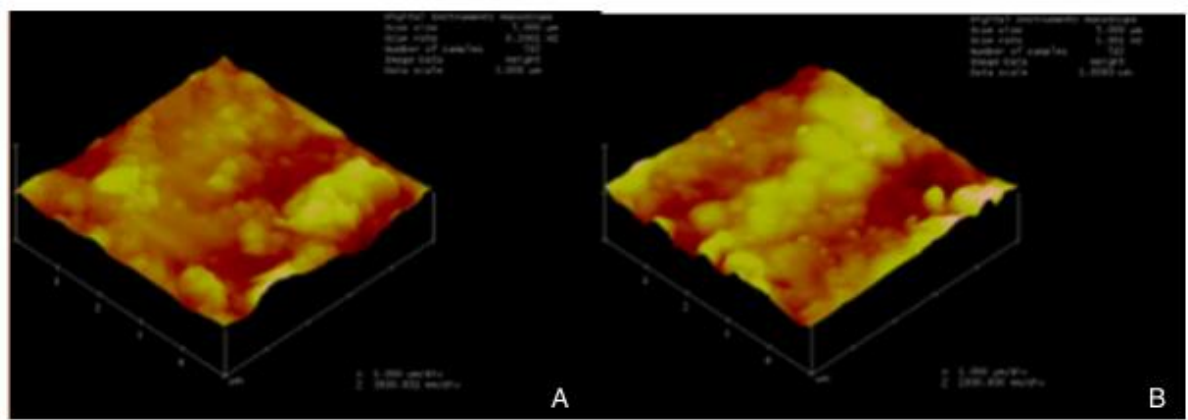

Figs. 4a-b AFM images of a) Control, non-conditioned $(45.8 \pm 20 \mu \mathrm{m})$, b) Tribochemical silica coated $(83 \pm 35.4 \mu \mathrm{m})$ zirconia surfaces. 